\title{
UN TEMPS SANS DEHORS : VALÉRY ET LA DANSE
}

Frédéric Pouillaude

\author{
Le Seuil | « Poétique »
}

2005/3 nº 143 | pages 359 à 376

ISSN $1245-1274$

ISBN 9782020687712

Article disponible en ligne à l'adresse :

https://www.cairn.info/revue-poetique-2005-3-page-359.htm

\section{Pour citer cet article :}

Frédéric Pouillaude, «Un temps sans dehors : Valéry et la danse », Poétique 2005/3 $\left(\mathrm{n}^{\circ} 143\right)$, p. 359-376.

DOI 10.3917/poeti.143.0359

Distribution électronique Cairn.info pour Le Seuil.

(C) Le Seuil. Tous droits réservés pour tous pays.

La reproduction ou représentation de cet article, notamment par photocopie, n'est autorisée que dans les limites des conditions générales d'utilisation du site ou, le cas échéant, des conditions générales de la licence souscrite par votre établissement. Toute autre reproduction ou représentation, en tout ou partie, sous quelque forme et de quelque manière que ce soit, est interdite sauf accord préalable et écrit de l'éditeur, en dehors des cas prévus par la législation en vigueur en France. Il est précisé que son stockage dans une base de données est également interdit. 


\section{Frédéric Pouillaude Un temps sans dehors: Valéry et la danse}

Qu'un art soit un art ne va nullement de soi. Encore faut-il qu'il soit identifié et reconnu pour tel. Mieux, assigné dans un réseau de différences et d'identités le reliant à l'ensemble des autres arts : pour reprendre la formule traditionnelle, dans un "système des Beaux-Arts ». La danse est massivement absente de tels systèmes. Art mineur et hybride, offert selon l'espace louche de la scène et de la fête, produisant des «objets » évanouis sitôt que montrés, la danse ne saurait prendre part au défilé de ce qui se nommait jadis "Beaux-Arts». Et lorsque le discours littéraire ou philosophique s'en empare, ce n'est généralement que pour confirmer une telle minorité. On ne parlera pas des œuvres produites par la danse, mais plutôt de l'expérience toute privée qu'elle procure, de ce que c'est que danser pour celui ou celle qui danse, hors la structure nécessairement duelle du théâtre et de la scène. Cet oubli des œuvres et du lieu où elles se donnent prolonge et justifie l'exclusion de la danse hors de l'"artisticité ": une expérience dont aucun produit ne se détache n'est sans doute pas un art. Et pourtant, cette absence d'œuvre va autoriser dans le discours une opération étonnante : la transformation immédiate de l'« en deçà » en "au-delà ", le passage du "sous-art " au "sur-art ", et, en un mot, l'élévation de la danse au transcendantal. Du fait même de sa minorité et de l'inconsistance de ses produits, la danse deviendra une sorte d'art originaire, un art d'avant l'éparpillement empirique des arts, un "art » où s'exhiberaient inentamées l'unité et la condition de possibilité de tous les arts constitués. C'est cette étrange identité du mineur et du transcendantal, cette articulation permanente de l'en deçà et de l'au-delà - selon nous caractéristique des discours théoriques sur la danse -, que nous souhaiterions mettre ici en évidence à partir de Valéry.

Trois textes forment l'essentiel des écrits consacrés par Valéry à la danse : un dialogue poético-socratique, L'Ame et la Danse' sion d'une prestation chorégraphique, Philosophie de la danse ${ }^{2}$; et un bref chapitre définitionnel extrait de Degas. Danse. Dessin, "De la danse " ${ }^{3}$. Quinze années séparent L'Ame et la Danse (1921) des deux derniers (1936). Si le premier texte laisse clairement voir le poids de l'héritage mallarméen ${ }^{4}$, les suivants semblent s'en éloigner fermement. De plus, le statut poétique et dialogique de L'Ame et la Danse, autorisant la coexistence de thèses multiples et la formulation d'intuitions n'ayant pas à fournir leur preuve, contraste avec le caractère ouvertement thétique et 
démonstratif des deux autres. Pour des raisons tenant aussi bien à la nature des textes qu'à celle de notre propos, nous parlerons ici essentiellement de ces derniers, n'ayant la plupart du temps recours au premier qu'à des fins de confirmation ou de complément.

Le style de ces deux textes, la tonalité qui s'en dégage immédiatement, comme à leur surface et en même temps au cœur de leur opération, tient en un certain mélange de frivolité et de grandiloquence. Non pas selon le partage attendu : un discours sérieux (philosophique) sur un objet léger (la danse). Mais exactement l'inverse: un discours assumant de part en part sa frivolité, à propos d'un objet dont Valéry soutient pourtant qu'il est le plus sérieux qui soit. Pour ce qui est de la frivolité assumée, disons qu'elle naît d'un excès de gravité feinte. Valéry prend un plaisir évident à surjouer le ton philosophique, à pousser d'un cran sa rhétorique ordinaire, et à l'abolir ainsi dans la comédie de son propre sérieux. La chose se laisse lire en partie dans les titres choisis : "De la danse " évoque à l'envi le thème latin et le traité scolastique, et "Philosophie de la danse " semble bien pompeux pour désigner un texte d’à peine dix pages qui ne fait somme toute que retracer un dire de circonstance. Valéry était-il à ce point appesanti par l'esprit de lourdeur pour commettre en toute naïveté de pareils intitulés? L'hypothèse semble peu probable. Ces textes sont-ils, à l'inverse, foncièrement ironiques, reproduisant depuis un feint sérieux les tours usuels de la méthode philosophique, les exhibant sur le mode réflexif et pince-sans-rire du pastiche? C'est ce qu'invitent à penser nombre de remarques, livrées au détour des pages, et thématisant justement, en son artifice même, la méthode philosophante ici employée :

Voilà bien de la philosophie, pensez-vous... Je le confesse... J'en ai mis un peu trop. Mais quand on n'est pas danseur; quand on serait bien en peine non seulement de danser, mais d'expliquer le moindre pas, on n'a de salut que dans quelque philosophie - c'est-à-dire que l'on reprend les choses de fort loin avec l'espoir de les faire sévanouir dans la distance 5 .

La façon d'un philosophe, son entrée en danse est bien connue... Il esquisse le pas de l'interrogation. Il joue son jeu. Il commence par son commencement ordinaire. Et le voici qui se demande : «Qu'est-ce donc que la danse? " [...] Mon philosophe en est là : hésitant sur le seuil redoutable qui sépare une question d'une réponse, obsédé par le souvenir de saint Augustin, rêvant dans la pénombre à l'embarras de ce grand saint : "Qu'est-ce que le Temps? Mais qu'est-ce que la danse?... » Mais la danse, se dit-il, ce n'est après tout qu'une forme de Temps, ce n'est que la création d'une espèce de temps, ou d'un temps d'une espèce toute distincte et singulière. Le voilà déjà moins soucieux : il a fait le mariage de deux difficultés ${ }^{6}$.

Réservons pour l'instant cet hymen de la Danse avec le Temps. Présenté comme association contingente, issu du trouble philosophique et de l'inévitable réminiscence augustinienne qui s'ensuit, il n'est pas sans évoquer la logique du lampadaire : ce n'est pas là que l'on a perdu ses clés, mais du moins l'on y voit plus clair ; en tout cas, on est moins soucieux. Cet hymen - on y viendra plus tard - consti- 
tue l'essentiel de ce que Valéry a à dire sur la danse. Mais dans l'immédiat, retenons ceci : ce qui se définit ici comme philosophique tient avant tout dans une certaine position d'extériorité du discours à l'égard de son objet, dans une certaine absence d'appui empirique, de fréquentation ou de connivence avec ce dont on parle. On est bien en peine d'expliquer quelque pas. Une seule opération demeure disponible : l'évanouissement de la chose dans la distance, son évaporation dans une configuration de discours réglée ailleurs, au loin. Telle serait la frivolité bien connue de la philosophie. Parole sous condition d'une suspension de tout savoir, elle ne peut s'en remettre qu'aux nervures dialectiques du langage, et à elles seules. Pour ce faire, elle dispose de quelques tours récurrents, de quelques pas déjà appris, de commencements ordinaires, indéfiniment répétables. Valéry, qui n’est pas parmi les moins lucides, reconnaît cette frivolité et l'assume. Sur le mode inévitable du pastiche ${ }^{7}$.

Pourtant, lorsqu'il est question de l'objet même, ou plus exactement de son statut, la frivolité de ton s'arrête net, laissant place à un sérieux qui n'est pas loin de la grandiloquence. Etant admis que l'objet est donné ordinairement comme de peu de poids, il s'agit bien ici de le réhabiliter, puisque nous, philosophes, jugeons nécessaire d'en parler. Ce ton de la réhabilitation ouvre Philosophie de la danse, aussi bien qu'il le ferme:

J'entre tout de suite dans mes idées, et je vous dis sans préparation que la Danse, à mon sens, ne se borne pas à être un exercice, un divertissement, un art ornemental et un jeu de société quelquefois; elle est chose sérieuse et, par certains aspects, chose très vénérable ${ }^{8}$.

J'ai voulu montrer comment cet art loin d'être un futile divertissement, loin d'être une spécialité se bornant à la production de quelques spectacles, à l'amusement des yeux qui le considèrent ou des corps qui s'y livrent, est tout simplement une poésie générale de l'action des êtres vivants 9 .

Encore une fois, réservons le terme de cette citation - l'énigmatique poésie générale de l'action - et notons simplement ce à quoi il s'oppose: la futilité du divertissement, la production de spectacles, l'amusement des yeux, bref, tout ce qui à certains égards constitue l'effectivité empirique de la danse en tant qu'art, en tant que production d'objets partageables, offerts depuis l'écart d'une scène. Car c'est bien sur les ruines de la scène et du spectacle que la réhabilitation aura lieu. En tant que production scénique, la danse est assurément vouée au mineur, au voisinage du cirque, de la fête foraine et des cracheurs de feu. On ne peut la considérer comme objet grave qu'en la décalant, qu'en l'inscrivant hors effectivité. De sorte que le sérieux attribué à l'objet va dans le même sens que la frivolité proclamée du discours; ils s'acheminent tous deux vers une même conséquence: une nécessaire mise entre parenthèses, voire un total oubli ou déni, de l'empiricité. Valéry ne cite à aucun moment le titre d'une ouvre ni le nom d'un artiste. Pas davantage, il ne prend en compte le fait que la danse se donne ordinairement sur la scène, dans l'horizon du spectacle. Et ce double déni est directement lié au ton adopté, à ce 
mélange inédit de frivole et de sérieux qu'il propose. Le sérieux de l'objet se conquiert contre l'empirie, et la frivolité du discours, son caractère philosophique surjoué, ne fait qu'autoriser à un autre niveau cet oubli. Un régime différent aurait été envisageable. Exactement inverse : un discours n'ayant pas peur d'être sérieux à propos d'un objet parfaitement léger. Disons, par provision, que cet autre discours fut exactement celui de Mallarmé dans Crayonné au théâtre.

Et c'est là que la position de Valéry étonne. A plus d'un titre. S'il ne fait guère de doute que ces textes sont encore dans une large mesure commandés par le souvenir de Mallarmé, il est frappant de voir comment Valéry s'y emploie cependant à démembrer l'héritage. Ce qui chez Mallarmé se donnait comme indissociable - la danse dans l'horizon de la scène - se retrouve chez Valéry parfaitement épars. Car le biais qu'adopte Valéry concernant la danse n'est pas lié à un désintérêt pour le spectacle en lui-même. Mais plutôt à une opération de disjonction. Valéry est autant fasciné que son maitre par l'espace scénique, dont il voit aussi bien que lui les enjeux politiques et religieux. Cette fascination se dit explicitement dans Mes théatres ${ }^{10}$, et l'on retrouve dans l'exercice quotidien des Cahiers un assez grand nombre de notes laissant entrevoir une théorie du spectacle fort élaborée et toute personnelle ${ }^{11}$. Bien plus, il semble que Valéry ait poussé d'un cran l'intérêt. Avec Amphion 12, "mélodrame " créé en 1931 sur une musique de Honegger et une chorégraphie de Fokine (le tout sous la houlette d'Ida Rubinstein et de ses Ballets); avec Sémiramis ${ }^{13}$, "mélodrame " créé en 1934 avec la même équipe, Valéry franchit un pas que Mallarmé s'était toujours gardé de faire : celui de la réalisation scénique. Là où Mallarmé tirait de la fascination pour le théâtre le fol espoir du Livre, qui devait précisément par l'écriture suppléer à la splendeur et à la ritualité de la scène, Valéry au contraire passe à l'acte, se faisant tout bonnement librettiste et collaborateur occasionnel des ballets Rubinstein ${ }^{14}$. Au risque, d'ailleurs, d'une certaine déception ${ }^{15}$.

Valéry sait donc que la danse peut avoir lieu au théâtre et que cette opération n'est pas nulle. Si la fréquentation de l'Opéra ne suffisait à lui imposer cette vérité, du moins peut-on supposer que l'autorité de Mallarmé y parvint. Il sait également qu'il existe des ballets et des chorégraphes, puisqu'il collabore avec ${ }^{16}$. Enfin, la notion de «spectacle" lui importe au plus haut point, comme l'atteste abondamment l'œuvre privée des Cahiers. Et pourtant, lorsqu'il parle et écrit publiquement sur la danse, il semble contraint d'oublier tout cela, de ne traiter la danse qu'hors la scène, en un énigmatique espace. Si l'on devait se risquer à rendre compte d'une telle disjonction, c'est encore une fois la double tonalité des textes qu'il s'agirait d'invoquer: le sérieux de la réhabilitation s'adossant à la frivolité du philosophique comme tel. La danse n'échappe au statut d'art mineur qu'en s'extrayant de l'insignifiance du spectacle. Et cela tombe bien, puisque jamais un discours philosophique ne s'abaisserait à penser cela, qui est l'empirie même.

C'est donc de philosophie qu'il va s'agir. D'où la caractérisation de l'objet comme essence, et l'inauguration de la réflexion par l'inévitable question «Qu'estce que... ?". "Qu'est-ce donc que la Danse? ", se demande notre philosophe. La Danse. Pas le ballet, ni le bal musette, ni le flamenco (dont pourtant il est censé 
parler un peu ${ }^{17}$ ), pas plus le menuet ou la farandole. Non, la Danse, essence une et intemporelle, située au-delà de toute détermination singulière, cachée derrière le chatoiement empirique des diverses gesticulations et posée comme à leur fondement. Ce qui donne accès à l'essence, on a coutume de l'appeler, en philosophie, définition. Et c'est bien d'une définition de la danse qu'il sera ici question, pouvant saisir toute danse en sa communauté d'essence. Or - une fois mises de côté les simplement nominales -, il existe deux grands types de définition. L'une procède par emboîtement de classes et restrictions successives. Pour parler comme Platon, par série de dichotomies. Pour parler comme Aristote, par genre et différence spécifique. Appelons cette définition taxinomique, et disons qu'elle consiste à isoler la bonne case au sein d'un système classificatoire global. L'autre n'a que faire de la classification et entend simplement montrer d'où la chose procède, d'où elle tient son être. Elle tente d'élucider l'essence par retour à l'origine, et fait le pari que cette origine peut s'entendre comme détermination essentielle. Appelons-la génétique. Disons alors, au risque de forcer un peu les textes, que "De la danse " met en œuvre une définition taxinomique, et Philosophie de la danse une définition génétique.

\section{Définition taxinomique, ou pourquoi Danser n'est pas Marcher}

"De la danse" s'ouvre par une définition en bonne et due forme. A première vue, par genre et différence spécifique:

La danse est un art des mouvements humains, de ceux qui peuvent être volontaires ${ }^{18}$.

Cette définition pose évidemment problème. Notamment par la différence qu'elle propose, trop vaste et trop restrictive à la fois. La catégorie de "volontaire " exclut nombre de faits de danse où la volonté semble précisément abolie: danses sacrées, danses "primitives", éventuellement danses de bal, où chaque fois l'antique nouage de la danse à l'extase et à la transe semble attesté. Valéry en est bien conscient, lui qui qualifie ailleurs la danse de "somnambulisme ${ }^{19}$ ». Différence trop restrictive, donc. Par ailleurs, et c'est l'évidence même, les mouvements volontaires accueillent bien autre chose que les dansants; des uns aux autres, il y a comme l'écart du genre et de l'espèce. Différence trop vaste, donc. A moins que la différence soit en réalité inscrite dans le vocable «art » qui forme le premier versant de la définition. Malgré l'apparence syntaxique, ce serait alors le second membre qui constituerait le genre - les mouvements volontaires -, et le premier la différence spécifique - «art ${ }^{20}$. Danse : ensemble de mouvements volontaires, dont le propre est de se donner comme de l'art. A supposer que l'on mette de côté la première objection (différence restrictive), il faudra encore tout le parcours de «De la danse " pour rendre compte de cette définition liminaire. 
Supposons que «mouvements volontaires» soit le genre. Il convient alors de tracer un premier partage, d'isoler une première différence, selon que la fin est ou non extérieure au mouvement. Parmi les mouvements volontaires, il y a ceux dont le télos est toujours projeté hors d'eux-mêmes, dans quelque objet à saisir ou quelque lieu à rejoindre ${ }^{21}$, et qui de ce fait sont toujours déjà achevés, déjà accomplis dans la représentation du but qui les anime ${ }^{22}$. D'autre part, il y a ceux qui sont à eux-mêmes leur propre fin, qui n'ayant ni terme ni objet n'ont pas d'évolution ni de conclusion prévisibles ${ }^{23}$. De ces derniers mouvements, on trouverait des exemples éminents dans les bonds et gambades de l'enfant, ou les sauts de l'animal qui s'ébroue. Encore loin de la danse, nous approchons cependant. Car une première détermination - temporelle - s'annonce ici essentielle. Selon l'ambiguïté bien connue du mot «fin», une activité sans télos doit également se comprendre comme activité in-finie. Etant à eux-mêmes leur propre fin, ces mouvements sont en droit illimités et ne trouvent leur achèvement que dans une modification extérieure qui les arrête:

[Ils] ne cessent que par quelque intervention étrangère à leur cause, à leur figure, à leur espèce ${ }^{24}$.

Cette différence temporelle, cette détermination temporelle de la différence, s'adosse à une certaine économie de la dépense. Les mouvements pratiques s'économisent dans la représentation d'une fin qui par avance les achève. Les mouvements gratuits ne peuvent que viser à une dissipation totale, dont le terme, en soi toujours repoussé, n'advient que par accident :

Au lieu d'être assujettis à des conditions d'économie, il semble, au contraire, qu'ils aient la dissipation même pour objet ${ }^{25}$.

Cette opposition du finalisé et du dissipatoire, du pratique et du gratuit, dans leur rapport différencié au temps, entraîne quant à la danse un motif constant. Puisque la Danse appartient au second genre, elle sera de par sa nature même in-finie. Elle ne pourra consister qu'en la perpétuation indéfinie d'un état, ne cherchant autre chose que sa propre prolongation. Ainsi, dans Philosophie de la danse:

La danse se passe dans son état, elle se meut dans elle-même, et il n'y a, en ellemême, aucune raison, aucune tendance propre à l'achèvement. Une formule de la danse pure ne doit rien contenir qui fasse prévoir qu'elle ait un terme ${ }^{26}$.

Autrement dit, la structure de la dépense ouvre, hors la téléologie finie de l'action, un nouveau rapport au temps. Et c'est dans une telle temporalité, in-finie parce que sans dehors, que la Danse va se déployer.

Cependant, tout n'a pas encore été dit avec la dichotomie entre mouvement pratique et mouvement gratuit. La Danse est loin de s'identifier à l'ensemble des mouvements de dissipation, et n'équivaut sûrement pas aux gestes de l'animal qui s'ébroue, expulsant son trop-plein d'énergie. Il faut, pour atteindre la danse même, 
introduire une seconde différence, cerner une nouvelle espèce au sein des mouvements gratuits. :

Mais il existe une forme remarquable de cette dépense de nos forces : elle consiste à ordonner ou organiser nos mouvements de dissipation ${ }^{27}$.

Telle est l'essence de la danse. Elle consiste en une certaine mise en forme de la dissipation; elle ordonne la puissance surabondante et construit en rythmes et figures l'élan informe de notre force vitale. Et ce qui se redonne avec cette dernière spécification, c'est très exactement le sens du vocable "art » lancé dans la définition liminaire. L' "art ", c'est assurément l'inutile et le gratuit ${ }^{28}$. C'est d'ailleurs ainsi que nous l'avions pris jusqu'ici, essayant de lire les mouvements de dissipation selon les termes de la définition. La dissipation, parce que déployée dans l'inutile, est amorce d'art. Mais l'art, c'est aussi bien, accompagnant ou contrebalançant cette inutilité première, une certaine puissance de configuration, une certaine élaboration qui, raffinant l'inutile, travaille à informer les rebuts de la vie pratique. De sorte que la danse est art des mouvements humains selon deux motifs bien distincts : par son appartenance à l'inutile, par sa puissance d'ordonnancement. Par là, elle se situe à la racine d'une ambiguïté qui travaille tout art. Elle met au jour, pour ce que l'on nomme "art", une exigence constitutive et contradictoire. D'un côté, une dissipation sans reste, au nom de laquelle s'ouvre l'inutile, de l'autre, une force de configuration capable de transformer la dépense en objet.

Cette tension, disons par anticipation qu'elle est celle-là même de l'œuvre, en tant que distincte aussi bien du produit que du rien. Elle est cette spécificité de l'œuvre - sa condition d'impossibilité peut-être - qui tente de se penser sous divers noms depuis Kant jusqu'à Blanchot. De la théorie du Génie à la thématique de l'absence d'auvre, se déploie une même thèse. C'est dans l'épreuve de sa propre impossibilité que l'œuvre, si elle est bien œuvre et non produit ou objet, peut trouver le gage de son existence. Pas de règles de l'art chez Kant, mais le Génie comme totale absence de préceptes, et par là l'impossibilité proclamée de ce qui en ressort ${ }^{29}$. Pas d'œuvre à proprement parler chez Blanchot, mais plutôt l'absence d'euvre comme condition et signification des résidus qui occasionnellement s'en dégagent ${ }^{30}$. Simplement, l'existence même des œuvres, leur stabilité rassurante et massive en forme d'objets, masque ce noyau d'impossibilité qui originairement les conditionne, occulte ce nouage à la dissipation pure qui d'un même mouvement barre et appelle leur existence. La Danse, par contre, produisant peu d'objets, nous livre la tension à l'état natif. Art d'avant l'art, art d'avant sa résorption en œuvre, et par là, travaillant aux conditions de possibilité de celle-ci. Bref, un art transcendantal. 


\section{Définition génétique, de l'excès de force à la poésie générale de l'action}

Une autre façon de saisir de l'essence, avons-nous dit, consiste à montrer d'où la chose procède, d'où elle dérive, et à penser cette origine comme détermination essentielle. La définition taxinomique de "De la danse " pouvait déjà se lire selon une telle logique. La Danse naîtrait de certains états corporels privilégiés, états de dissipation et de dépense, où le corps cesse d'être le simple organon de l'action pour se viser lui-même à travers des mouvements sans ni fin ni objet. La Danse ne serait que la complexification et l'ordonnancement de ce sol originaire, dont elle ne se détacherait jamais vraiment. C'est une telle lecture génétique que nous offre Philosophie de la danse.

Le terme d'où procède la Danse n'est autre que la Vie même, dans sa plus grande généralité. De ce terme, il faut dire plus précisément que la Danse se déduit. Ce régime de la déduction implique deux choses : que la Danse soit déjà en germe dans la vie même, pour ainsi dire analytiquement comprise en elle, et que cependant elle s'en détache suffisamment pour se différencier :

C'est que la Danse est un art déduit de la vie même, puisqu'elle n'est que l'action de l'ensemble du corps humain; mais action transposée dans un monde, dans une sorte d'espace-temps, qui n'est plus tout à fait celui de la vie pratique ${ }^{31}$.

Le premier motif qui vient à l'appui de la déduction s'entend sur le mode de l'identification restrictive. La Danse n'est que... Et ce que désigne cette restriction, c'est, valant aussi bien pour la Danse que pour la vie, une certaine absence de spécification et de supplément techniques. Il n'y a pas d'outil pour danser. A certains égards, pas non plus pour vivre. Dans les deux cas, il n'y a pas de domaine privilégié sur lequel l'activité aurait à se concentrer et à se spécialiser. Ce qui est donné à l'une et à l'autre, ce n'est que "l'action de l'ensemble du corps humain", dans toute son ampleur et sa nudité. Travaillant à même la généralité de la vie, prise dans la même étoffe, antérieure à toute spécification technique, la Danse ne fait que prolonger nos gesticulations les plus simples. Pourtant, elle se donne simultanément comme écart absolu. Elle se détache du monde des mouvements pratiques, de cet espace-temps de la praxis, où l'anticipation du but signale toujours déjà l'achèvement du mouvement. La Danse semble alors entretenir avec la vie un rapport parfaitement contradictoire. Elle est aussi bien excroissance vitale, sécrétion spontanée du corps, que subversion du lien à l'utile. Remarquons cependant que, lorsque la Danse se détache, c'est déjà d'une vie spécifiée et restreinte, d'une vie justement qualifiée de pratique. Y aurait-il alors, en deçà ou au-delà de celle-ci, une autre vie, une vie comme telle, une vie nue? Il semble que ce soit le cas. Et cette vie nue, d'où la Danse est censée dériver, Valéry la caractérise précisément comme surabondance, comme surplus de forces et de sensations dont on ne sait que faire: 
L'homme s'est aperçu qu'il possédait plus de vigueur, plus de souplesse, plus de possibilités articulaires et musculaires, qu'il n'en avait besoin pour satisfaire aux nécessités de son existence [...]. Nous avons trop de puissances pour nos besoins. [...] Nous pouvons exécuter une foule d'actes qui n'ont aucune chance de trouver un emploi dans les opérations indispensables ou importantes de la vie ${ }^{32}$.

Et c'est là qu'intervient le second motif. Si la danse se déduit de la vie même, sans doute est-ce parce qu'elle partage la même généralité, la même absence de spécification technique. Mais c'est également, et plus simplement, parce que la vie l'appelle directement, parce que la vie n'est en elle-même qu'un surplus qui attend d'être dépensé. De cet excès sur soi qui caractérise tout vivant (car le propre de l'homme n'est que de s'en apercevoir), il faut dire que la Danse est à la fois conséquence immédiate et développement dérivé : conséquence "immédiate », puisqu'elle se joue sur le même terrain et que c'est sans médiation qu'elle dissipe le trop-plein; développement "dérivé ", puisque c'est aussi bien d'un art qu'il s'agit, lequel implique un minimum d'artifice et d'arbitraire, venant détourner et reconfigurer à son profit la surpuissance vitale. La Danse, art déduit de la vie même. Avec cette déduction - ou origine - de la Danse, ce n'est rien de moins qu'une certaine ambiguïté humaine qui se trouve exhibée à l'état natif. A la fois simple vivant ancré dans son milieu et animal assez singulier pour accorder un véritable prix à l'inutile ${ }^{33}$, l'homme trouve dans la Danse l'image de sa propre ambivalence, le point de conversion où la nature verse depuis ses forces propres du côté de l'artifice, et où le vivant, s'échappant à lui-même, invente de nouvelles configurations, aussi arbitraires qu'inutiles, et cependant inscrites dans le mouvement de la vie même. C'est ce passage que la déduction valérienne donne à penser, en forme de nœud contradictoire. La Danse est au plus près de la vie, en ce qu'elle est faite du même "élan ", et au plus loin, en ce qu'elle est déjà échappement et rupture essentielle - sorte de nature seconde où ne cesserait pourtant pas de s'attester la première :

Cette seconde nature est ce qu'il y a de plus éloigné de la première, mais il faut qu'elle lui ressemble à si méprendre ${ }^{34}$.

Ce nœud contradictoire, ce jeu du proche et du lointain, on peut à l'arrivée le déployer en forme de chronologie fictive, selon une logique proprement génétique :

J'ai essayé de vous communiquer une idée assez abstraite de la Danse, et de vous la présenter surtout comme une action qui se déduit, puis se dégage de l'action ordinaire et utile, et finalement s'y oppose ${ }^{35}$.

Mais l'on s'aperçoit vite que cette généalogie excède de très loin son objet. S’y discerne comme en germe un certain discours anthropologique sur la possibilité ou l'origine de l'art, entendue dans sa plus grande généralité. La Danse est ce moment où l'homme verse dans l'inutile pour le raffiner. En général. Avant toute spécification de domaine ou d'objet. Avant toute détermination technique. Et c'est 
pourquoi il faut y voir bien plus que ce que l'on y discerne ordinairement: non pas un futile divertissement, non pas une vulgaire production de spectacles, mais rien de moins que la possibilité générale de l'art s'exhibant à même la vie. Cette version génétique - ou diachronique - de l'élévation de la Danse au transcendantal (la Danse comme point d'origine de tout art) doit accueillir une version synchronique. Il faut montrer comment l'origine est encore à l'œuvre dans le présent de chaque art, et comment chaque art n'est que le cas particulier d'une certaine idée générale de la Danse, entendue comme art transcendantal, comme Art d'avant l'éparpillement empirique des arts :

Ce point de vue d'une très grande généralité (c'est pourquoi je l'ai adopté aujourd'hui) conduit à embrasser beaucoup plus que la danse proprement dite. Toute action qui ne tend pas à l'utile, et qui, d'autre part, est susceptible d'éducation, de perfectionnement, de développement, se rattache à ce type simplifié de la danse, et, par conséquent, tous les arts peuvent être considérés comme des cas particuliers de cette idée générale $[\ldots]^{36}$.

On retrouve ici l'articulation de la dépense et de l'ordonnancement, de l'inutile et du perfectionnement, déjà à l'œuvre dans la définition taxinomique. Si l'«art " se définit synthétiquement comme le perfectionnement de l'inutile, la Danse en constitue alors l'emblème général. Les différents arts ne font que décliner cet emblème selon la diversité des sens, des matières et des techniques. Mais un second motif intervient. Si bien qu'il nous faut reprendre la citation :

[...] tous les arts peuvent être considérés comme des cas particuliers de cette idée très générale, puisque tous les arts, par définition, comportent une partie d'action, l'action qui produit l'œuvre, ou bien qui la manifeste.

Un poème, par exemple, est action, parce qu'un poème n'est qu'au moment de sa diction : il est alors en acte. Cet acte, comme la danse, n'a pour fin que de créer un état; cet acte se donne ses lois propres; il crée, lui aussi, un temps et une mesure du temps qui lui conviennent et lui sont essentiels: on ne peut le distinguer de sa forme de durée. Commencer par dire des vers, c'est entrer dans une danse verbale $^{37}$.

On s'aperçoit alors que ce dont la Danse est le modèle ne concerne pas l'entièreté de chaque art, mais seulement ce qui dans chacun relève de l'actualité, ou encore du présent. Cette actualité n'est pas celle de l'œuvre achevée. Elle réside au contraire dans ses marges. Pour reprendre les catégories usuelles : en amont, dans l'action qui la produit, en aval, dans celle qui la manifeste. Dans chaque cas, si les arts s'adossent à la Danse, c'est parce que l'activation de l'œuvre oblige à en excéder la ferme substantialité, parce qu'il faut un peu "danser " autour pour la faire se lever. L'œuvre n'est possible qu'en vertu de ce qui lui est le plus étranger, qu'en vertu de ce qui de soi-même la désoeuvre. La nécessité - peut-être fantasmée, du moins ici scandée - d'un recours à l'acte, d'un détour par l'actualisation de ce qui sans cela ne resterait que virtuel, évaporé dans les songes de l'œuvre à venir, ou à 
demi mort dans les traces de l'œuvre passée, conduit donc à voir dans la danse - actualité sans œuvre - le présent vivant de tout art.

Selon cette double version de l'élévation au transcendantal (diachronique/synchronique), nous pouvons désormais comprendre l'énigmatique formule lancée par Valéry, cette poésie générale de l'action des êtres vivants au nom de laquelle la Danse devait échapper à la vaine frivolité du spectacle. Sur le mode diachronique, elle désigne ce moment où l'action vitale en vient à se poétiser, à travailler dans l'inutile et à le raffiner, moment dont dérive tout art. Sur le mode synchronique, elle révèle comme en creux ce qu'est tout poème véritable. Non pas un ensemble de traces, mais plutôt, en deçà de la solidité de l'œuvre, la réactivation indéfinie d'un état. On sait bien que pour Valéry le poème n'existe qu’à être lu, qu’à être réactivé dans le présent vivant d'une énonciation. On sait également qu'il est en lui-même infini, toujours à remettre sur le métier, ne trouvant jamais de terme, sinon, au même titre que la Danse, par accident ${ }^{38}$. Et, dans cette articulation de la Danse et du Poème, c'est encore une fois un certain rapport au temps et au présent qui se trouve engagé.

\section{Description du «danser » : un corps abîmé en lui-même}

Ce qui se confirme à un autre niveau.

Car, en marge des définitions et des déductions, un régime de discours bien différent apparaît, où Valéry assigne à l'écriture une tâche excédant de fort loin la nécessité artificielle du concept. Au-delà des agencements taxinomiques et des dérivations abstraites, il convient de donner à voir. Il convient d'exhiber, dans l'intuition et l'évidence sensibles, la chose même, et par là d'en reproduire un tant soit peu la chair. Valéry serait donc plus empiriste qu'on ne croyait.

Encore faut-il saisir ce qui au juste est décrit. "Une danseuse qui danse »! Quoi de plus normal, dira-t-on? En même temps, au-delà de la détermination sociologique qui fait qu'en ces années 1930 danser est encore nécessairement l'affaire d'une "danseuse ", quoi de plus abstrait? On ne décrira pas tel ou tel ballet, ni même telle ou telle danseuse dansant un rôle du répertoire. On décrira simplement "une danseuse qui danse ", comme si cette dernière opérait dans une indétermination et une généralité telles que, quels que soient ce qu'elle danse et la façon dont elle le danse, c'est toujours plus ou moins la même chose qui à l'arrivée se produit. Bien pire, on ne décrira pas même la "danse " comme telle, comme ensemble de gestes, de rythmes et de figures déterminés, mais seulement le «danser ", comme expérience subjective. Non pas un produit, mais une expérience vécue, par essence dérobée au regard. Car il semble bien, à lire Valéry, que jamais rien ne se détache de la danseuse, que rien ne s'y montre véritablement, sinon les indices d'un vécu tout privé, qu'il s'agira de décrire, vaille que vaille.

Ce vécu tout privé, ce "danser ", consiste en une expérience du détachement, ou, pour reprendre une expression déjà lancée, de l'échappement. Le corps dansant 
est un corps "échappé " ${ }^{39}$. Il s'extrait du monde ordinaire des travaux et des tâches. Il se construit une demeure à soi, toute faite d'immanence, où les mouvements s'engendrent d'eux-mêmes, selon leurs règles propres. De façon négative, le détachement se comprend donc comme rupture et opposition. C'est ainsi que l'entendait notre définition génétique :

Ce détachement du milieu, cette absence de but, cette négation des mouvements explicables $[\ldots]$, tous ces traits sont décisivement opposés à ceux de notre action dans le monde pratique et de nos relations avec lui ${ }^{40}$.

De façon plus positive - et c'est là qu'intervient la description -, il s'entend comme enfermement et construction, plus exactement comme enfermement construit. On pourrait ici multiplier les citations :

Il apparaît que cette personne qui danse s'enferme, en quelque sorte, dans une durée qu'elle engendre, toute faite d'énergie actuelle, toute faite de rien qui puisse durer ${ }^{41}$. [...]

Il observe que ce corps qui danse semble ignorer ce qui l'entoure. Il semble qu'il n'ait affaire qu'à soi-même [...]. Oui, ce corps dansant semble ignorer le reste, ne rien savoir de ce qui l'environne. On dirait qu'il s'écoute et n'écoute que soi. [...] C'est donc bien que la danseuse est dans un autre monde, qui n'est plus celui qui se peint de nos regards, mais qu'elle tisse de ses pas et construit de ses gestes ${ }^{42}$.

Ne voyant rien de ce qui l'environne, la danseuse s'enferme. Malgré son apparente frénésie d'espace, elle ne fait, par ses mouvements sans dehors, que se replier en elle-même, que se claquemurer dans un corps qui, horizon absolu de son présent, élimine toute extériorité. Cet enfermement se comprend également comme immédiate réflexivité. Sphère d'intériorité pure, la danseuse sécoute. Elle ne fait même que cela. Elle passe son temps à s'écouter. Ou plutôt, elle semble faire de l'écoute de soi le fondement même du temps, presque sa forme a priori. L'intime attention est le milieu et la condition même de la durée qu'elle engendre. A condition d'inverser les termes, Kant, Bergson et Husserl ne sont guère loin!

- Voilà bien de la philosophie...

- Je le confesse... J'en ai un peu trop mis.

Et cependant, par l'articulation de la réflexivité et du temps que la danse exhibe en suspendant le monde, c'est bien de philosophie qu'il s'agit, et plus précisément, d'une certaine figure de la conscience reproduite à même le corps. Entre la corporéité dansante et la pensée se pensant elle-même, surgit un parallèle frappant ${ }^{43}$. Entre la conscience qui, pour mieux s'assurer d'elle-même, refuse de donner son assentiment au monde, et le corps qui se soustrait au jeu ordinaire de l'utile pour mieux jouir de soi, il y a une commune structure d'épochè, une identique mise entre parenthèses de l'extériorité. Et depuis cette suspension, la même immanence se dégage, la même sphère - conscience ou corps propre - $s$ 'instaure, où rien ne se donne, sinon soi-même s'auto-affectant, dans le temps. On imagine à quel point notre philosophe est ravi. Il est en terrain connu : 
Le philosophe exulte. Point d'extériorité! La danseuse n'a point de dehors... Rien n'existe au-delà du système qu'elle forme par ses actes, système qui fait songer au système tout contraire et non moins fermé que nous constitue le sommeil, dont la loi tout opposée est l'abolition, l'abstention totale des actes ${ }^{44}$.

Singulière épochè, tout de même! Epochè corporelle qui oscille entre le rêve et le cogito, et qui, se pensant comme extrême attention, entretient néanmoins d'étranges liens avec le sommeil et l'abolition de toute conscience ${ }^{45}$ ! "Sphère de vie lucide et passionnée ${ }^{46}$ ", la danse donne à voir un corps à la fois englouti et extralucide, parfaitement "aliéné", en ce que son appartenance à un autre monde peut se lire comme "envoûtement " et "possession ", et parfaitement «libéré", en ce que son activité repose sur un mouvement de "déprise " qui le rend à lui-même (et le fait échapper à l'hétéronomie du geste pratique). Etrange puissance d'un corps qui joue au plus fin, qui oscille perpétuellement entre l'écoute lucide de soi et l'automatisme de la transe! C'est là que ne manque pas d'intervenir le thème du somnambulisme :

La danse lui apparaît comme un somnambulisme artificiel, un groupe de sensations qui se fait une demeure à soi, dans laquelle certains thèmes musculaires se succèdent selon une succession qui lui institue son temps propre, sa durée absolument sienne ${ }^{47}$.

Mais il conviendrait justement de penser ce qui fait l'artificialité de ce somnambulisme-là, de comprendre en quoi ce dernier est toujours construit et institué, se distinguant de l'enfermement dans le sommeil simple. Valéry ne nous y aide guère. Il nous indique seulement que la construction se fait dans et par le temps. La danseuse institue son temps propre; elle construit des sensations de durée absolument siennes. Mais elle n'opère jamais que sur elle-même. Fermée à tout dehors, son " œuvre " n'est qu'une vie intérieure, légèrement modifiée :

Vie intérieure, mais celle-ci toute construite de sensations de durée et de sensations d'énergie qui se répondent et forment comme une enceinte de résonance ${ }^{48}$.

Prise dans son enceinte, sphère d'auto-affection pure, la danseuse ne connait que le temps. Par les sensations qu'elle se donne en s'y enfermant, elle ne fait que reproduire l'articulation originaire du Soi et de la temporalité, que rejouer, en l'amplifiant et la raffinant, une très intime pulsation qu'il est convenu d'appeler "vie intérieure ». Il faut alors - au risque du paradoxe - tirer une conséquence : la danseuse ne sait rien de l'espace. Conséquence pleinement assumée par Valéry, notamment dans « De la danse » :

Nous avons déjà dit, que dans ce genre de mouvements, l'Espace n'était que le lieu des actes : il ne contient pas leur objet. C'est le Temps à présent qui joue le grand rôle ${ }^{49}$.

La Danse, à force de n'être que plis et replis sur soi-même, doit être au final pensée comme pure indifférence à l'espace. 
Cette conséquence n'était pas inéluctable. Car l'enceinte fut un bref moment entaillée. Il y eut pour la danseuse, le temps d'un instant, un autre objet que soimême. Et il nous faut reprendre une citation, tout à l'heure volontairement tronquée :

Il observe que ce corps qui danse semble ignorer ce qui l'entoure. Il semble bien qu'il n'ait affaire qu'à soi-même et à un autre objet, un objet capital, duquel il se détache ou se délivre, auquel il revient, mais seulement pour y reprendre de quoi le fuir encore...

C'est la terre, le sol, le lieu solide, le plan sur lequel piétine la vie ordinaire, et procède la marche, cette prose du mouvement humain ${ }^{50}$.

La terre, le sol, est bien cet autre objet où s'annonçait l'extériorité de l'espace. Par là, on pouvait envisager une autre forme de construction pour qualifier la danse, une construction d'espace, de lieux, de directions, etc., et pas simplement de durées intimes. Mais l'extériorité de la terre va se trouver d'emblée annulée. Sans doute la terre est-elle, dans cette prose du mouvement qu'est la marche, le support de l'extériorité. Mais, dans la poésie de la danse, elle est immédiatement intégrée à l'intimité de la sensation; elle s'y transforme en simple sentiment de poids, en ensemble différencié de pesanteurs vécues, avec lesquelles la danseuse tisse son enceinte. Cette métamorphose poétique de la terre en sensations pondérales s'indique par un fait simple et constant. La danseuse valérienne a toujours les yeux fermés:

Elle est tout entière dans ses yeux fermés, et toute seule avec son âme, au sein de l'intime attention ${ }^{51}$.

Dans la clôture de ses yeux fermés, la danseuse n'a plus affaire à l'espace que comme poids. Sorte de trou noir spontané, elle absorbe la terre; elle l'allège en un vécu sans dehors qui, s'alignant sur la pure inextension du sentir, en vient à nier toute localité. Plus vraiment d'espace, donc. Rien qu'une terre entrelacée de temps ${ }^{52}$.

Attardons-nous encore quelques instants sur ces "yeux fermés ". A travers eux, c'est également l'ensemble du régime descriptif adopté par Valéry qui se trahit. Plus exactement, une certaine situation de regard très simple et très complexe à la fois, se nommant "voyeurisme». Valéry regarde danser une danseuse. Il en a même les yeux exorbités. La danseuse, quant à elle, ferme les siens, toute perdue au sein de l'intime attention, engloutie dans un corps qui ne voit plus rien. Elle ne s'adresse nullement à Valéry. Disons simplement qu'elle s'abandonne, qu'elle se laisse passivement regarder, se sachant elle-même bien ailleurs. Pas l'once ici d'une signification adressée, d'un discours même latent destiné par un Je à un Tu. Rien ne se détache de la danseuse qui puisse relever d'une quelconque intention envers autrui. La danseuse ne dit rien, et à personne. A la place de ce discours inexistant, demeure une chose très pauvre et très générale, nullement faite pour être vue: la jouissance d'un corps s'absorbant en lui-même, « la pure et immédiate violence de 
l'extrême félicité ${ }^{53}$ ". La pauvreté de cette jouissance conditionne aussi bien la généralité des descriptions (quelle que soit la danse, c'est toujours le même phénomène qui est en jeu) que le dispositif sur lequel elles s'appuient (le voyeurisme). Cette chose qui ne s'adresse à personne, et qui du même coup ne manque pas d'induire chez autrui l'irrépressible désir de la voir, d'avoir les yeux plantés sur cela même qui n'est pas fait pour être vu, ne s'exhibe que selon un dispositif précis : qu'on puisse la regarder sans être soi-même vu voyant. Telle est la seconde fonction des yeux fermés. Non seulement assurer la clôture de la jouissance, mais également dessiner comme en creux la place de celui qui y portera les yeux, la place du voyeur.

On comprend alors que l'intérêt pour la danse devienne, selon une équation propre à Valéry et pourtant bien banale, pure fascination pour le sexe de la femme, et que ce sexe, entendu selon la métaphore maritime de la Méduse, résume à lui seul toute la situation de regard, tout l'entrecroisement d'yeux fermés et ouverts - médusés - où Valéry insère son discours. Un semblant de relation s'instaure alors. Selon la pauvre logique du désir et de la prostitution :

Jamais danseuse humaine, femme échauffée, ivre de mouvement, du poison de ses forces excédées, de la présence ardente de regards chargés de désir, n’exprima l'offrande impérieuse du sexe, l'appel mimique du besoin de prostitution, comme cette grande Méduse, qui, par saccades ondulatoires de son flot de jupes festonnées, qu'elle trousse et retrousse avec une étrange et impudique insistance, se transforme en songe d'Eros; et tout à coup, rejetant tous ses falbalas vibratiles, ses robes de lèvres découpées, se renverse et s'expose, furieusement ouverte ${ }^{54}$.

Du sérieux de la réhabilitation à l'excitation solitaire de la pornographie court un identique fil continu: l'oubli de la scène et du théâtre, l'oubli de ce lieu où l'on s'adresse à qui regarde, et où, depuis cette adresse même, se dégage un tiers objet. Une œuvre. Ou à défaut, un sens.

\section{Université Lille-III}

\section{NOTES}

1. L'Ame et la Danse, in numéro spécial de la Revue musicale du 1er décembre 1921: Le Ballet au XIX siècle, Ed. de la Nouvelle Revue française, repris dans Euvres, t. II, Paris, Gallimard, «Bibliothèque de la Pléiade », 1960, p. 148-176.

2. Philosophie de la danse, conférence prononcée à l'Université des Annales le 5 mars 1936, publiée dans Conferencia, $1^{\text {er }}$ novembre 1936, reprise dans Euvres, t. I, Paris, Gallimard, "Bibliothèque de la Pléiade », 1957, p. 1390-1403.

3. Degas. Danse. Dessin, Paris, Ambroise Vollard, 1936, repris dans Euvres, t. II, Paris, Gallimard, «Bibliothèque de la Pléiade », 1960, p. 1163-1241. Sauf indication contraire, c'est toujours dans l'édition de la Pléiade que nous citerons désormais Valéry. 
4. Dans une lettre adressée à Louis Séchan (août 1930) pour le remercier de l'envoi de son ouvrage, $L a$ Danse grecque antique (Paris, E. de Boccard, 1930), Valéry ne manque pas de reconnaitre un tel poids quant à l'écriture de L'Ame et la Danse: "Je n'aurais jamais projeté d'écrire sur la danse à laquelle je n'avais jamais sérieusement pensé. D'ailleurs, j'estimais, - et je l'estime encore, - que Mallarmé avait épuisé le sujet en tant qu'il appartient à la littérature. Cette conviction m’a fait d'abord refuser la commande de la Revue musicale. D'autres raisons m’ont déterminé à l'accepter. Ce que Mallarmé avait prodigieusement écrit est alors devenu une condition singulière de mon travail. Je ne devais ni l'ignorer ni l'épouser. J'ai pris le parti de faire figurer, parmi les interprétations diverses que donnent de la danse les 3 personnages, celle dont l'énoncé et l'incomparable démonstration par le style se trouve (sic) dans les Divagations " (Lettres à quelques-uns, Paris, Gallimard, 1951, p. 191). Ce personnage qui, dans L'Ame et la Danse, représente Mallarmé, c'est Phèdre s'opposant à Eryximaque, opposition résumée par Socrate en ces termes: "O mes amis, je ne fais que vous demander ce que c'est que la danse; et l'un et l'autre paraissez respectivement le savoir; mais le savoir tout à fait séparément! L'un me dit qu'elle est ce qu'elle est, et qu'elle se réduit à ce que voient ici nos yeux [Eryximaque, le médecin]; et l'autre tient très ferme qu'elle représente quelque chose, et donc qu'elle n'est point entièrement en elle-même, mais principalement en nous [PhèdreMallarmé, le poète] ", L'Ame et la Danse, in CEuvres, op. cit. t. II, p. 165.

5. Philosophie de la danse, in Euvres, op. cit., t. I, p. 1394. Nous soulignons.

6. Ibid., p. 1395-1396.

7. L'Ame et la Danse, faisant dialoguer Socrate, Eryximaque et Phèdre au terme d'un festin trop abondant, se donne déjà explicitement comme un pastiche du banquet platonicien, l'idée du Beau se levant à même la grâce légère et dénudée des corps dansants, venus charmer et solliciter les convives appesantis. Valéry, d'une certaine façon, ne fait que réécrire un Banquet où danseuses et citharistes n'auraient pas été d'emblée exclus de l'assemblée philosophique des convives, un Banquet où Musique et Danse, loin de détourner l'âme de son bien propre, seraient au contraire, dans la matérialité de leur présence, les ferments spirituels de l'investigation dialectique sur le Beau. Outre Platon, il faudrait bien sûr songer au Banquet de Xénophon, où les figures des danseurs viennent ouvrir et clore le dialogue, Socrate vantant au plus haut point les bienfaits d'une activité et reconnaissant la mettre lui-même en pratique.

8. Philosophie de la danse, in CEuvres, op. cit., t. I, p. 1391. Nous soulignons.

9. Ibid., p. 1402.

10. Article paru le 19 septembre 1942 dans Les Dernières Nouvelles, repris dans Euvres, op. cit., t. I, p. 1791-1794.

11. Essentiellement, dans l'édition du CNRS : VI, 150; VI, 508; IX, 659; XIV, 776; XV, 164; XV, 187 ; XVI, 648 ; XVII, 426 ; XVII, 758 ; XIX, 11-12 ; XX, 510-511 ; XXII, 496 ; XXIII, 901 ; XXIII, 901 922; XXIV, 11-12; XXIV, 16; XXIV, 315; XXIV, 565; XXIX, 672.

12. Amphion, mélodrame créé à l'Opéra de Paris le 23 juin 1931, repris dans Euvres, op. cit., t. I, p. 166178. Voir également, Histoire d'Amphion, in Euvres, op. cit., t. II, p. 1277-1283.

13. Sémiramis, mélodrame créé à l'Opéra de Paris le 11 mai 1934, repris dans Euvres, op. cit., t. I, p. 182-196.

14. Henri de Régnier ne manque pas de remarquer, dans sa critique d'Amphion pour Le Figaro du 26 juin 1931, tout ce qui dans une telle tentative rattache Valéry à Mallarmé : "Nous avons bien souvent, M. Valéry et moi, entendu notre cher Stéphane Mallarmé exposer ses idées sur les combinaisons possibles qu'offrirait au théâtre l'alliance du vers, de la musique et de la danse. Sans doute, M. Valéry s'est souvenu de ces conversations, en composant son "mélodrame" d'Amphion. C'est une très intéressante expérience qu'il a tentée et dont Mme Ida Rubinstein lui a apportée l'occasion ", Dossier de presse "Amphion », Bibl. de l'Opéra de Paris-BNF.

15. Sur ce point, voir la lettre à Sully Peyre (1933), où Valéry justifie en ces termes la publication d'Amphion: "J'ai publié ce texte - à tort peut-être. Mais l'exécution de l'ouvrage fut telle que j'ai pensé par là établir pour quelques-uns mon intention. Je n'ai pu malheureusement imposer mes vues, qui étaient les plus simples du monde, et j'ai eu l'ennui d'assister à un ballet russe quand j'avais conçu une sorte de cérémonie religieuse. L'indiscipline et l'incohérence ou la fantaisie ont régné ", Lettres à quelques-uns, op. cit., p. 209-210.

16. Toujours au régime des collaborations, il faudrait mentionner les préfaces de Valéry pour Serge Lifar (Préface à Serge Lifar à l'Opéra Défini par Paul Valéry Parlé par Jean Cocteau Vécu par Serge Lifar, Paris, T. de Champrosay, 1943, et Avant-propos aux Pensées sur la danse, Paris, Bordas, 1946). Enfin, les Cahiers nous apprennent qu'un projet de ballet fut envisagé pour Lifar en 1941, sous le titre provisoire d'" Harmoniques " ou de "Figures» (op. cit., XXIV, 95) : «Je cherche un sujet de ballet pour Lifar - (affaire). Je ne 
trouve rien aujourd'hui qui convienne. Mon sujet des "Ames" est trop difficile pour être traité au vol. Je pense à une trame assez rigoureuse sur laquelle je broderais un sujet. Les harmoniques. Figures.» Ces informations, ainsi qu'une bonne partie de celles qui précèdent, sont issues du précieux ouvrage d'Huguette Laurenti, Paul Valéry et le théâtre, Paris, Gallimard, 1973.

17. La conférence de Valéry précédait la prestation chorégraphique de Mme Argentina, danseuse flamenca.

18. Degas. Danse. Dessin, «De la danse», in Euvres, op. cit., t. II, p. 1170.

19. Il est vrai "artificiel» (Philosophie de la danse, in CEuvres, op. cit., t. I, p. 1398). Mais il nous semble - comme nous essaierons de le montrer plus loin - que Valéry a justement les plus grandes difficultés à penser cette artificialité-là.

20. Si effectivement " art des mouvements humains " était le genre et "volontaires " la différence, cela impliquerait qu'il y ait un "art des mouvements involontaires", art dont on ne voit pas très bien ce qu'il serait sinon encore une fois la danse, selon l'une de ses manifestations possibles.

21. "Il s'agit d'atteindre un lieu ou un objet, ou de modifier quelque perception ou sensation en un point déterminé ", Degas. Danse. Dessin, «De la danse », in CEuvres, op. cit., t. II, p. 1170.

22. "Sa détermination contenait son extermination; on ne pouvait ni le concevoir, ni l'exécuter, sans la présence et le concours de l'idée d'un événement qui en fût le terme", ibid.

23. «D'autres mouvements dont aucun objet localisé n'excite, ni ne détermine, ni ne puisse causer et conclure l'évolution ", ibid.

24. Ibid.

25. Ibid. Nous soulignons.

26. Philosophie de la danse, in Euvres, op. cit., t. I, p. 1399.

27. Degas. Danse. Dessin, "De la danse ", in CEuvres, op. cit., t. II, p. 1171.

28. "Le caractère manifeste d'une œuvre d'art peut se nommer inutilité ", Notion générale de l'art, in Euvres, op. cit., t. 1, p. 1405.

29. Critique de la faculté de juger, $\$ 46$.

30. L'Entretien infini, Paris, Gallimard, 1969, et notamment "L'absence de livre».

31. Philosophie de la danse, in Euvres, op. cit., t. I, p. 1390. Nous soulignons.

32. Ibid., p. 1391-1932.

33. "L'homme est cet animal singulier qui se regarde vivre, qui se donne une valeur, et qui place toute cette valeur [...] dans l'importance qu'il attache à des perceptions inutiles et à des actes sans conséquences vitales ", ibid., p. 1393.

34. L'Ame et la Danse, in Cuvres, op. cit., t. II, p. 156.

35. Philosophie de la danse, in Euvres, op. cit., t. I, p. 1400.

36. Ibid.

37. Ibid.

38. Et c'est précisément pourquoi la Danse, dans son opposition à la Marche, peut servir de modèle à la Poésie dans son opposition à la Prose. Sur ce point, voir Poésie et pensée abstraite, in Euvres, op. cit., t. I, p. 1329-1330.

39. Voir la lettre à Séchan déjà citée : "La danse est le type de l'échappée. »

40. Philosophie de la danse, in Euvres, op. cit., t. I, p. 1399. Nous soulignons.

41. Ibid., p. 1396. Nous soulignons.

42. Ibid., p. 1397-1398.

43. A certains égards, ce parallèle est l'objet direct de L'Ame et la Danse, dans son intitulé même. Ainsi, par exemple (Euvres, op. cit., t. II, p. 171-172) : «Et le corps qui est ce qui est, le voici qu'il ne peut plus se contenir dans l'étendue! - Où se mettre? - Où devenir? Cet Un veut jouer Tout. Il veut jouer à l'universalité de l'âme! Il veut remédier à son identité par le nombre de ses actes! Etant chose, il éclate en événements! - [...] Ainsi le corps qui est là, veut atteindre à une possession entière de soi-même et à un point de gloire surnaturelle..."

44. Philosophie de la danse, in Euvres, op. cit., t. I, p. 1398.

45. "Rêve, rêve, mais rêve tout pénétré de symétries, tout ordre, tout actes et séquences!", L'Ame et la Danse, in Euvres, op. cit., t. II, p. 154.

46. Philosophie de la danse, in Euvres, op. cit., t. I, p. 1390.

47. Ibid., p. 1398. Nous soulignons.

48. Ibid., p. 1399-1400.

49. Degas. Danse. Dessin, "De la danse", in Euvres, op. cit., t. II, p. 25. 
50. Philosophie de la danse, in Euvres, op. cit., t. I, p. 1397. Nous soulignons.

51. L'Ame et la Danse, in Euvres, op. cit., t. II, p. 23.

52. «Elle semble d'abord, de ses pas pleins d'esprit, effacer de la terre toute fatigue, et toute sottise... Et voici qu'elle se fait une demeure un peu au-dessus des choses, et l'on dirait qu'elle s'arrange un nid de ses bras blancs... Mais, à présent, ne croirait-on pas qu'elle se tisse de ses pieds un tapis indéfinissable de sensations?... Elle croise, elle décroise, elle trame la terre avec la durée ", ibid., p. 160. Ou encore, p. 171 : "Comme il détruit furieusement, joyeusement, le lieu où il se trouve [...]."

53. Ibid., p. 170. Rappelons au passage que L'Ame et la Danse s'achève précisément sur une "petite mort ", sur l'évanouissement d'Athikté dans l'extrémité du plaisir, et sur son retour progressif à la vie : «PHÈDRE : Elle tourne, elle tourne... Elle tombe! SOCRATE : Elle est tombée! PHÈDRE : Elle est morte... [...] PHÈDRE : Qu'a-t-elle dit? SOCRATE : Elle a dit quelque chose pour soi seule. ERYXIMAQUE : Elle a dit : Que je suis bien! [...] SOCRATE : D'où reviens-tu? ATHIKTé: Asile, asile, ô mon asile, ô Tourbillon! J'étais en toi, ô mouvement, en dehors de toutes les choses...", ibid., p. 174-176.

54. Degas. Danse. Dessin, « De la danse », in CEuvres, t. II, p. 1173. 